\title{
Investigating educators' perspectives towards systems thinking in chemistry education from international contexts
}

Alisha R. Szozda ${ }^{1}$, Kathryn Bruyere ${ }^{1}$, Hayley Lee ${ }^{1}$, Peter G. Mahaffy ${ }^{2}$, Alison B. Flynn ${ }^{1 *}$

${ }^{1}$ Department of Chemistry and Biomolecular Sciences, Faculty of Science, University of Ottawa, Ottawa, Ontario, Canada

${ }^{2}$ Department of Chemistry and the King's Centre for Visualization in Science, The King's University, Edmonton, Alberta T6B 2H3, Canada

*Corresponding author. Email: alison.flynn@uottawa.ca

\section{Abstract}

Systems thinking in chemistry education (STICE) has been proposed as an approach that could better equip students with abilities to connect their chemistry knowledge with other disciplines, with the skills needed to tackle complex global issues. However, educational change in chemistry is a complex effort that involves many interconnected factors that enable or hinder chemistry educators' adoption of new pedagogical approaches. Using an adapted version of the TeacherCentered Systemic Reform (TCSR) model, we investigated factors that connect with chemistry educators' willingness and ability to implement a STICE approach in their courses. We surveyed a group of 56 secondary and post-secondary chemistry educators from ten different countries, to capture chemistry educators' perspectives towards a STICE approach. Through thematic analysis of responses, we found that educators' willingness and ability to implement STICE is influenced by their knowledge, beliefs, experiences, contextual and personal factors. We discuss specific aspects of the reform model that experts and administrators can address to reduce barriers to implement and engage with STICE. We also highlight future chemistry education research that is needed to explore specific aspects of educators' perspectives and STICE more broadly.

\section{Keywords}

Systems thinking in chemistry education (STICE), chemistry education, educators' perspectives, instructional change, qualitative, questionnaire 


\section{Abstract Graphic}

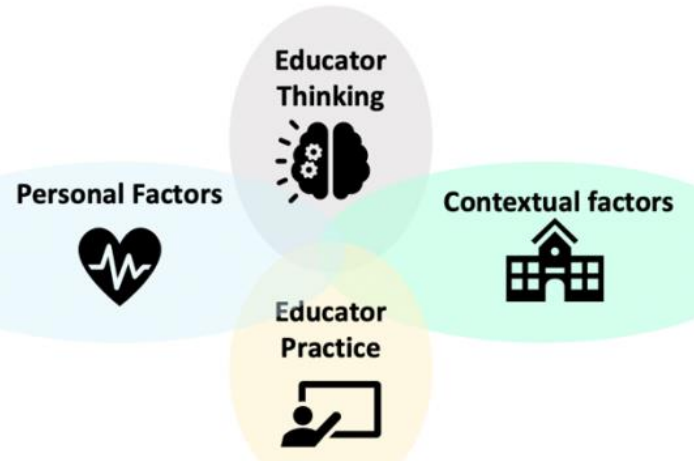

Chemistry is critically involved in tackling complex challenges, such as those prioritized with the United Nations' Sustainable Development Goals (SDGs), ${ }^{1,2}$ which include climate change and good health. ${ }^{3-6}$ However, current approaches in chemistry education often lack the connections to other disciplines and global issues, particularly in large enrolment courses and on assessments. ${ }^{7}$ In many courses, facts are learned in silos where emphasis is placed upon detailed descriptions of fundamental, yet narrow, principles. ${ }^{8-10}$ Consequently, learning discrete, unconnected facts out of context limits learners' abilities to apply knowledge and skills in new contexts, such as understanding how chemical processes impact and are impacted by societal, economic, political, and environmental factors. ${ }^{3,8,10}$

Building the capacity to achieve the SDGs and expectations of a $21^{\text {st }}$ century workplace requires evidence-based approaches to postsecondary STEM education. Recent compelling international calls have been made to reorient chemistry education using a systems thinking (ST) approach. ${ }^{7}$ ST has been proposed by chemistry educators as part of an approach to chemistry education that could bridge the disciplines and more effectively educate the next generation of scientists and prepare citizens for their roles on a rapidly changing planet. $2,63,63$

Research on ST in other disciplines suggests there would be strong benefits for introducing systems thinking in chemistry education (STICE). ${ }^{11-15}$ However, rigorous educational research is needed to determine effects and impacts of STICE approaches. ${ }^{3}$ Moreover, educators would need to be willing, able, supported, and rewarded in making such profound changes to their courses.

While there may be benefits and opportunities from implementing STICE, there are also challenges and risks. If ST approaches are to be successfully integrated in chemistry education, educators' perspectives and contexts must be deeply understood so that their needs can be addressed. These aspects play critical roles in educators' decisions to advance instructional change, based on extensive research at the secondary and postsecondary levels (e.g., educators' ability to make course or program changes). ${ }^{21-23}$ Additionally, beliefs held by educators about teaching and learning and their own abilities influence their adoption of new pedagogy. ${ }^{24,25}$

There are currently few ST teaching resources available and limited methods for assessing or measuring ST competencies. ${ }^{3,16,17}$ None of these assessment methods have been validated and 
are not designed to easily implement in large classes, such as first year chemistry university courses. ${ }^{3}$ Beyond individual teaching resources at the course level, ST approaches in chemistry courses need to be aligned with curricula at institutional and national levels, and international education standards. ${ }^{18-20}$ The present study is designed to investigate educators' perspectives about and knowledge of STICE, as to date, little is known about this critical group's perspectives.

\section{Goals and Research questions}

The overarching goal of this project is to investigate and prioritize the current actions and future research needed to help chemistry educators practically enact ST approaches. In this study we addressed the following research question: What are educators' knowledge about and perspectives on implementing STICE?

To conduct this study, we created and released a questionnaire to capture chemistry educators' perspectives globally towards a STICE approach. The findings from this questionnaire have two purposes: (1) to guide the creation of potential solutions to overcome educator barriers to determine the feasibility of the ST approach in different contexts and (2) inform future actions to address educator systemic change and additional CER that is needed to increase efforts of chemistry educators implementing STICE.

\section{Theoretical Framework}

We adapted the Teacher-Centered Systemic Reform (TCSR) Model to address the implementation of ST by chemistry educators. ${ }^{24}$ This model positions educators at the center of educational reform by placing emphasis on educators' thinking and interconnecting their knowledge and beliefs across three domains which are, (1) contextual factors, (2) personal factors, and (3) educator experience (Figure 1. ). For educators to enact a ST approach, educational reform efforts need to consider the interconnectedness between domains in the model. 


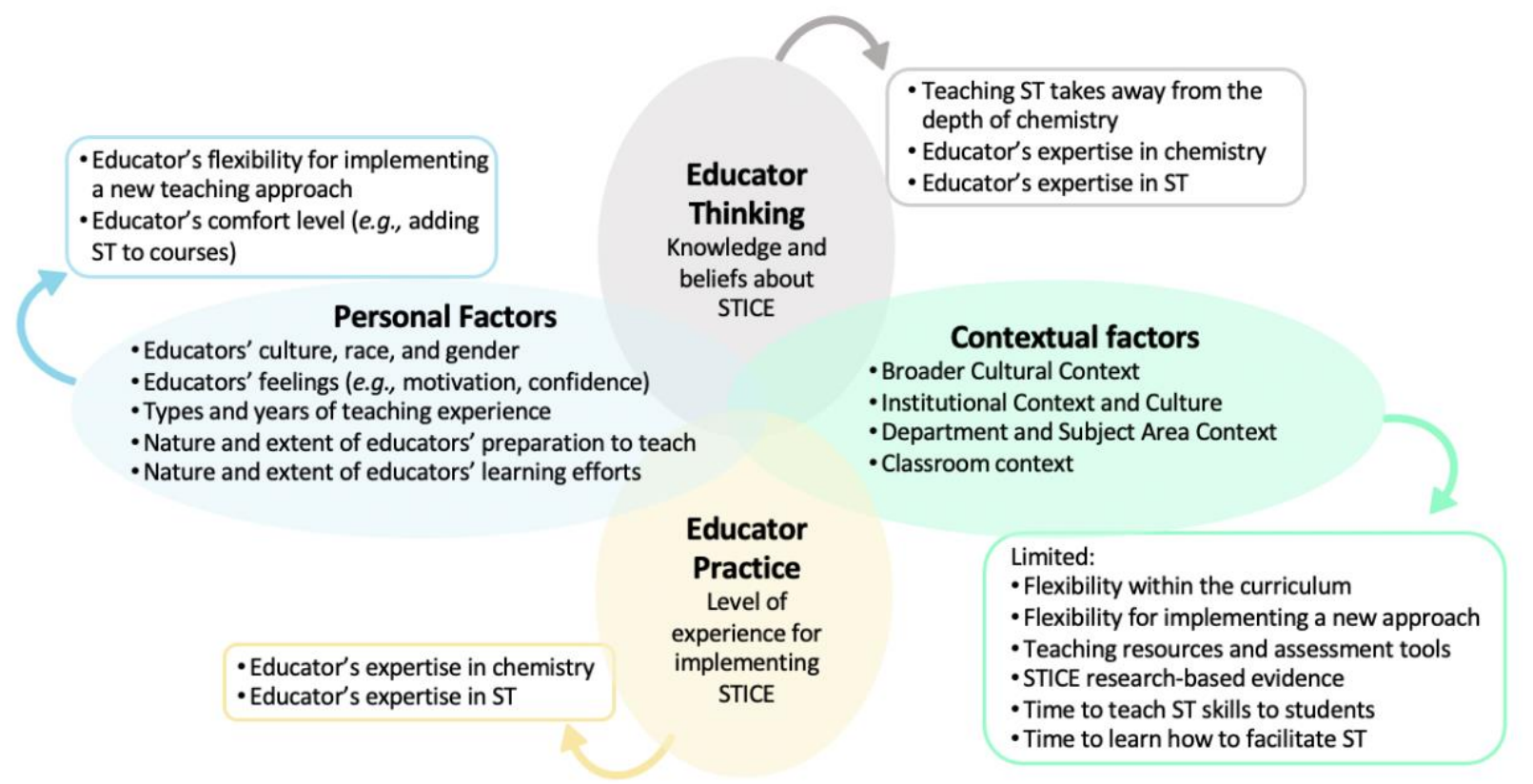

Figure 1. A STICE perspective of the Teacher-Centered Systemic Reform (TCSR) model (opaque ovals), aligned with potential STICE educator and educational barriers (outlined boxes) that we probed in the study.

\section{Methods}

\section{Instrument for data collection}

We developed a three-part questionnaire to capture educators' perspectives. Part 1 of the questionnaire used a combination of Likert-scale questions and open-ended questions aimed to: identify chemistry educators' current knowledge of STICE, identify how much flexibility educators have for implementing new teaching approaches (e.g., flexibility related to structural constraints, policies imposed by institution, departmental expectations), identify how much educators are willing to implement a ST approach, and determine potential student and educator barriers that may prevent educators from practically enacting this approach. We evaluated eleven educator/educational barriers aligned with the four factors of the TCSR model, that have been previously proposed by educators (Figure 1).

Part 2 investigated participants' experiences with STICE using open ended questions. We provided an operational definition and characteristics of STICE and asked educators to choose a category that best represented their experience with STICE. $8,27,28$ The options were: (1) I have not implemented ST into the course(s) I teach, (2) I have implemented ST into the course(s) I teach, or (3) I have implemented ST principles into the course(s) I teach. Categories 2 and 3 were designed to distinguish educators who explicitly (2) and implicitly (3) implement STICE. The categories were designed to guide participants to answer several open-ended questions related to their experience and perspectives on STICE based on the category chosen, requiring a written response. Educators who had experience with STICE were asked to identify which aspects of STICE they incorporate into their courses. We further explored how STICE is being taught, practiced, and assessed in their courses, when they started implementing STICE into 
their course(s), resources they use, resources they need, and how they overcome barriers associated with ST.

Part 3 of the questionnaire included demographic questions related to teaching (e.g., institution at which they teach, educational role, years of teaching, courses they teach), gender, culture, and race (details in Supplementary Information).

\section{Participants}

Fifty-six chemistry educators from ten different countries participated; they represented a combination of high school and postsecondary educators who identified as a woman, man, or non-binary, and teach a range of chemistry courses. This research received approval from the University of Ottawa's Office of Research Ethics and Integrity (\#H03-20-5585).

\section{Data Analysis}

The data qualitative analysis involved transcribing and analyzing the open response data in detail, using well-established educational research methods (details in SI). From that analysis, key themes emerged. We addressed the validity of the findings using well-established methods. 29,30

The quantitative analysis was carried out for the Likert scale questions related to knowledge, flexibility, willingness to implement STICE, years of teaching experience, experience with STICE and the student and educator/educational barriers questions. We used a Spearman's rank correlation test to determine any relationships among these factors. ${ }^{31}$ We conducted Kruskal Wallis tests to determine whether there was a statistically significant difference in the average extent of educator/educational barriers between the experience groups. In the following sections, we describe the main findings, starting with the key themes that emerged.

\section{Results and Discussion}

\section{Theme 1: Knowledge, experience, beliefs, personal and contextual factors}

\section{influenced educators' willingness to implement STICE}

Most participants (93\%) gave a neutral to positive response about their willingness of integrating STICE into their course(s) (Figure 2). A full 55\% of these participants were only somewhat likely to integrate STICE into their course(s), making us suspect that barriers or beliefs were affecting their ability or desire to implement STICE. No participants chose "not at all likely", which is a positive finding for the future of STICE adoption. 


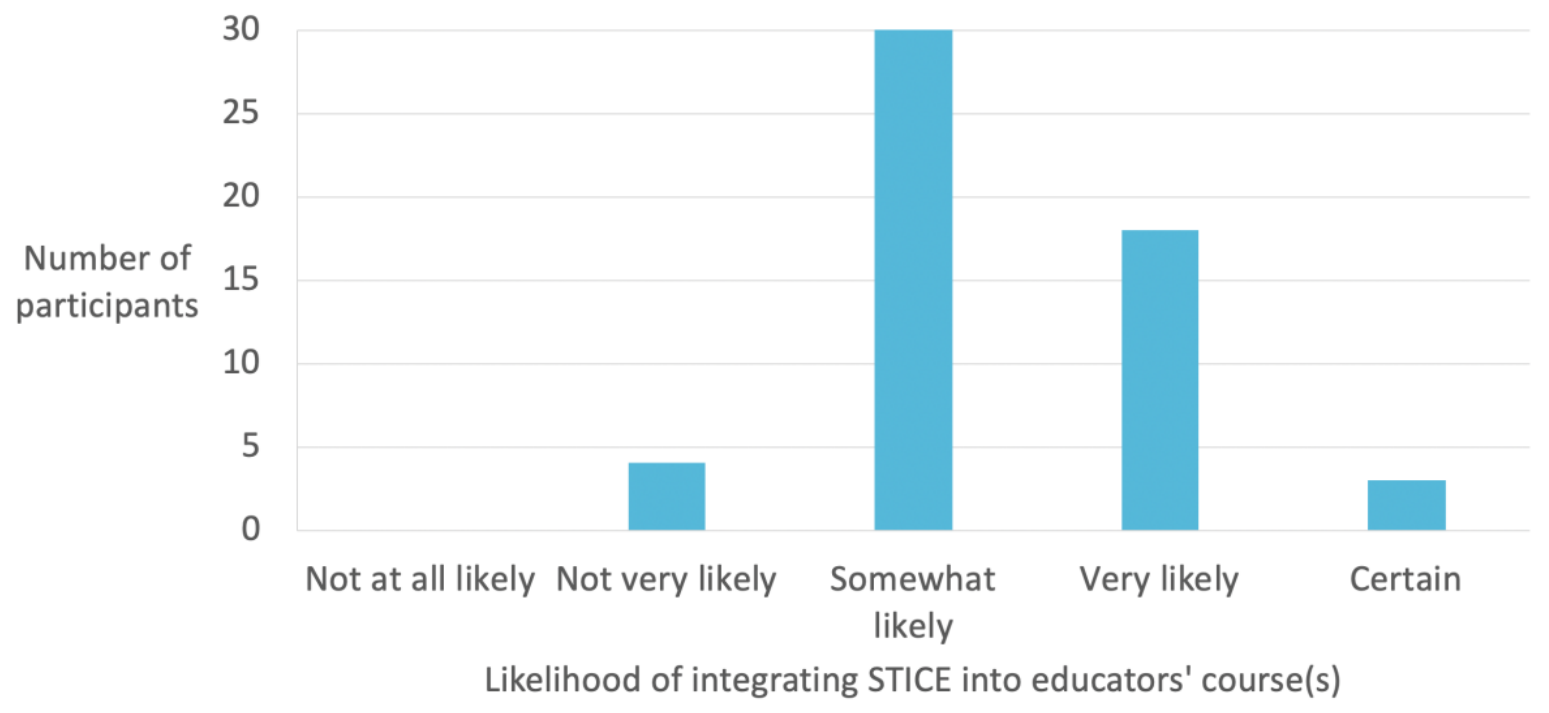

Figure 2. Educators' likelihood of integrating STICE into their course $(s)(N=55)$

Five categories of factors influenced educators' willingness to implement STICE: knowledge of STICE, experience with STICE, beliefs about STICE, and contextual and personal factors (Figure 3). Contextual factors had the largest influence on educators' willingness to implement STICE. Participants (55\%) indicated that their willingness would depend on: availability of teaching resources, curriculum coverage, research-based evidence, and time constraints to learn and implement STICE (Figure 3). Time and curriculum coverage limitations have also been recently emphasized as main limitations to implement STICE by other researchers, highlighting the substantial need for change in these areas. ${ }^{32,33}$

For successful adoption of STICE, there needs to be increased efforts for educational change at the departmental, institutional, and administrative levels (e.g., departmental STICE training, institutional policy changes, restructuring of curriculum, and assessments) as participants most frequently reported those four contextual factors as potential barriers to implement STICE.

Reward and promotion practices have also been concrete ways in which institutions incentivize educators' choices in their educational efforts. ${ }^{34}$

Educators were likely to implement STICE due to their experience, beliefs, and feelings towards STICE. Seven participants mentioned they have created or are creating a plan to implement STICE into their courses or they are already implementing STICE. These planned or existing experiences correspond to the high willingness to implement responses in the Likert scale question (Figure 2). 


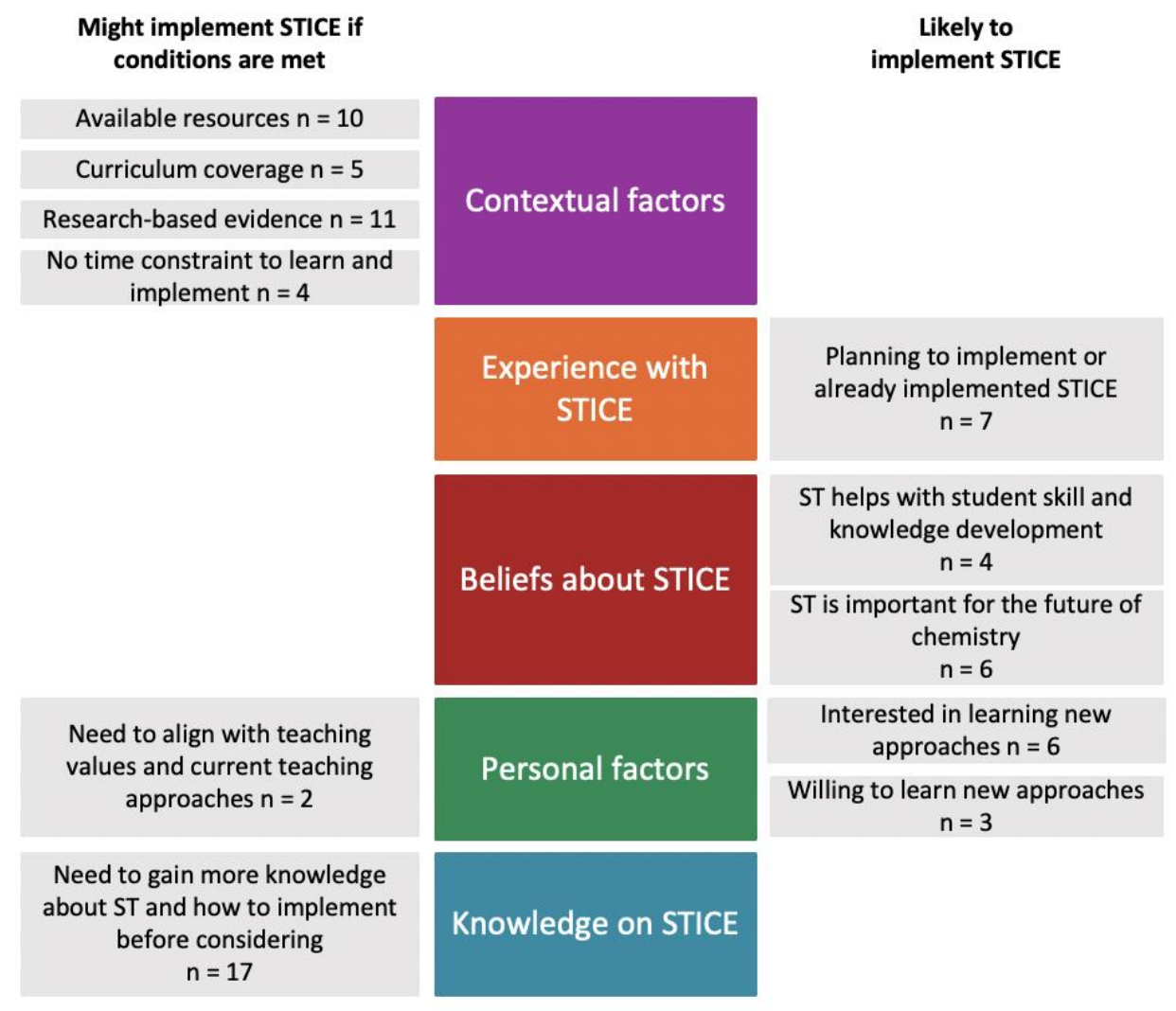

Figure 3. Factors that influence educators' willingness to implement STICE.

Educators had two common categories of beliefs about why they would be willing to implement STICE: (1) ST helps with student skill and knowledge development and (2) STICE is important for helping shape the future of chemistry and society (SI, Table 2). When participants were asked, "Do you think it is important for educators to implement a ST approach to their courses? Why or Why not?", the same beliefs emerged with the addition of another common belief that ST increases student engagement for learning chemistry (SI, Table 2). All educators who had one of these beliefs reported having experience with implementing STICE, which suggests that the importance of STICE and its benefits may emerge through their teaching experiences. Support of these findings are represented by recent publications from other STICE researchers who have investigated chemistry educator perspectives and have obtained similar results. ${ }^{32,35}$ While these findings represent positive perspectives on STICE, not all educators' beliefs were positive about the importance of implementing STICE. For example, educators believe there are issues with implementing STICE due to curriculum requirements and time constraints, both aligning with the contextual factors previously identified.

Educators also believe that there is a certain course level that is appropriate for implementing STICE. Educators gave two main reasons: limitations of students' skills and knowledge levels and the curricular change required by ST. Specifically, educators believe it would be difficult to implement STICE at an institution without every educator doing it. If an educator was the only one to implement STICE into their course within their department, they were concerned about how implementing STICE would affect other colleagues who depend on 
the pre-requisite knowledge for subsequent courses they teach and how it would impact students learning in upper-level courses. Such a situation commonly arises in first year chemistry courses (i.e., general chemistry).

To better understand educators' beliefs, future research is needed to (1) investigate when ST should be implemented in students' educational careers, along with the specific benefits and challenges it might provide for students at each course level and (2) determine if all educators are required to implement STICE in a program or at an institution for it to be effective.

Educators' feelings (personal factors) for trying new teaching approaches corresponded to higher willingness to implement STICE. Some educators were interested in learning about new teaching approaches $(n=6)$ and were willing to try new approaches $(n=3)$. On the other hand, two educators mentioned they would likely integrate STICE if the approach aligned with their teaching values or current teaching approach. This finding aligns with aspects of STICE that educators want to learn more about (e.g., how STICE can be integrated in current teaching approaches) (SI, Figure 4).

Educators' role-another personal factor-had an influence on educators' ability to implement STICE $(n=9)(S I$, Table 5). Generally, educators who were the only instructor for their course reported to have more flexibility for implementing new approaches compared to educators who taught one section of a large enrollment course or co-taught courses. This finding suggests that certain teaching contexts and roles may be viewed as a greater barrier to implement new approaches due to the collaborative nature of the job and the requirement that all instructors teaching that course may need to have similar views on how they plan on teach the content. Future research is needed to investigate how STICE can be effectively implemented in different teaching contexts that require educators to have similar perspectives.

Social pressure is an implicit and hidden factor that also emerged from analysis, affecting educators' flexibility to implement new teaching approaches (SI, Table 5). Specifically, social pressure from colleagues was reported to restrict educator's freedom to implement new approaches. Research on change in universities and colleges has demonstrated that systemic change occurs when a concerted effort is made by senior leaders (i.e., Deans, Department Chairs) in an institution to motivate their colleagues and obtain buy-in for change. ${ }^{36,37}$

In comparison to other predictor variables that were investigated through the questionnaire (i.e., flexibility, experience with STICE), knowledge of STICE was the only factor that showed a correlation between educators' willingness to implement, based on Spearman's rank correlation test, $r_{s}=0.457, p<0.001$, bias corrected accelerated $95 \%$ confidence interval $[0.184,0.674]$, a medium effect size (SI, Table 3). ${ }^{38}$

Seventeen participants indicated they would consider implementing STICE if they had more knowledge about STICE and/or how to implement STICE, which is consistent with educator's reported low level of knowledge of STICE (SI, Figure 2). Most participants were interested in learning ways to effectively implement and integrate STICE in a course or classroom and how to integrate STICE with other teaching pedagogies. Additionally, educators wanted specific examples and applications of STICE being implemented and assessed by chemistry instructors along with teaching and learning resources and assessment tools and examples to integrate 
STICE into their course(s). Assessment tools and teaching resources were the most frequently reported resources educators would need to implement STICE. Based on the efforts to date that have aimed to infuse ST approaches in chemistry education, our results indicate there is a need for professional development opportunities focused on building educators understanding of ST perspectives and demonstrating how to facilitate and integrate ST approaches to chemistry courses. ${ }^{3}$

Experts and policy makers may find the adoption of educational change models to be helpful in guiding future direction of the STICE. ${ }^{21,26,36}$ Specifically, in order for educators to change their willingness to adopt STICE approaches, efforts need to be made to increase educators' knowledge of STICE, minimize contextual factors primarily at the administrative level, and help align ST approaches with educators' values and current teaching approaches (personal factors).

Theme 2: Educators have experience with STICE without knowing or specifically calling it ST

While there was a range of STICE definitions from participants, six important characteristics of STICE emerged from their definitions: (1) ST is a holistic approach/ involves big picture thinking, (2) ST involves interconnections between concepts/topics/disciplines, (3) ST is an approach that applies chemistry to other contexts, (4) ST involves looking at variables that cause behaviours and outcomes,(5) ST involves understanding and analyzing complex systems, and (6) STICE recognizes that systems are embedded in chemistry.

Comparing educators' definitions and level of knowledge of STICE with their chosen category of experience, we found that educators may have experience with STICE without knowing about STICE or calling it systems thinking. Fifteen participants who reported little or no knowledge about STICE had no experience with implementing STICE, while sixteen participants within both knowledge groups (no or little knowledge) reported they had implemented STICE or implemented ST principles into the course(s) they teach (Figure 4). Figure 4 shows one participant self-reported thorough knowledge on STICE and no experience with implementing STICE. This participant may have had no experience with STICE due to their current role as a graduate student, which may limit their ability to implement STICE into a course. 


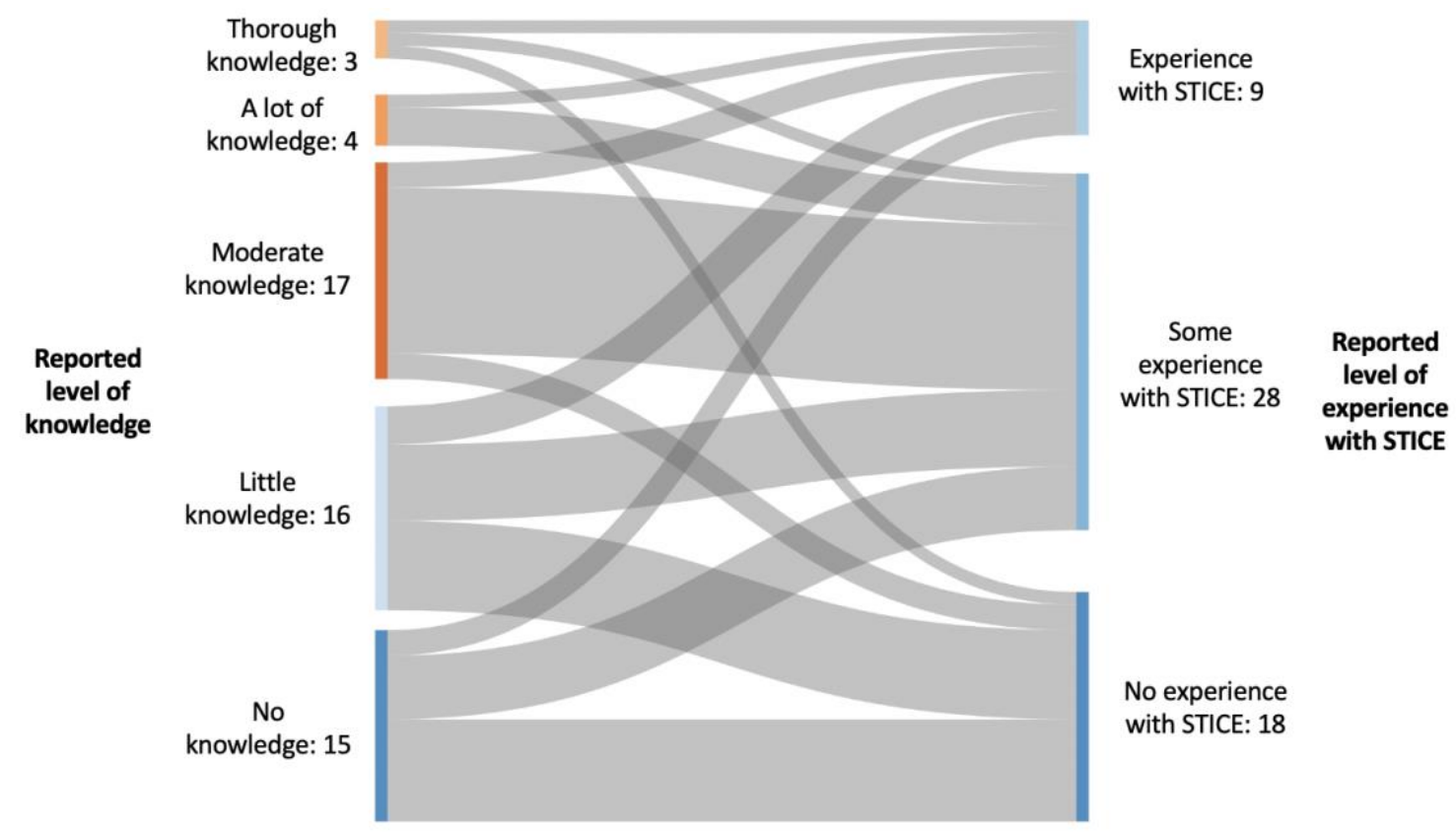

Figure 4. Educators' self-reported knowledge of STICE before receiving a STICE definition and educators perceived experience after receiving STICE definition.

Considering that over half of the participants claimed they had little to no knowledge on STICE, participants may not have known the extent of ST or never heard of ST but have been implicitly embedding ST principles and characteristics into their teaching pedagogy. For example, one participant recognizes they implement ST in one of their courses but realized they had never called it ST.

"Although I've never called it systems thinking (or thought of it as something different), it seems to be what I've been doing in this one course."

If chemistry educators were to become more knowledgeable on STICE, they will need to understand how to make changes to their current teaching approaches to better encompass a ST approach. Future research on STICE will be needed to determine the extent of adaptation made to chemistry educators current teaching pedagogy when ST approaches are integrated with the curriculum and program standards. Therefore, STICE educational change may be easier to achieve than expected as chemistry educators may not need to make huge modifications to their current teaching approach due the overlap of ST characteristics.

= Theme 3: Some chemistry educators implement limited aspects of STICE when teaching chemistry in context

We were able to confirm our hypothesis, "educators may have experience with STICE without knowing or calling it ST", by looking at how educators describe how they implement or how they plan to implement ST into their course(s).

Four categories emerged from participants' responses: (1) 54\% emphasized specific characteristics of STICE (e.g., focus on holism/bigger picture when teaching chemistry, teach causal behaviours of a system, and teach chemistry contexts within real-world context), (2) 18\% 
gave general ways to incorporate STICE into learning (e.g., STEM projects, curriculum, designed and applied activities), (3) 14\% use a different teaching approach to incorporate aspects of ST, and (4) $22 \%$ don't explicitly state how they implement STICE.

Thematic analysis revealed that educators primarily implement aspects of STICE in the following ways, (1) teaching chemistry with emphasis on societal and/or environmental context, (2) incorporating real world connections and applications of chemistry into their teaching, or (3) giving an example how connections to real life applications are taught in their chemistry course(s). These ways are aspects of STICE, but miss some important aspects needed to truly achieve a STICE approach (Figure 5).

Participants' responses show they incorporate real world connections of chemistry into their course(s) and report that they incorporate other characteristics of STICE into their course(s) but don't explicitly describe how they incorporate these other characteristics. This poses the question, "How do we know if educators are using a context-based approach rather than a STICE approach?".

\section{Systems Thinking}

- System as a whole

- Relationship between parts

- Causal variables

- Behavior over time

- Examining and analyzing complex systems
Context-based learning

- Students engage in high level interaction community

- Addressing chemically important concepts

- Using chemistry-specific language

- Building on prior knowledge

How educators in this study consider they are implementing ST

Figure 5. Similarities and differences of system thinking approaches and context-based learning. ${ }^{28}$ [Permission to adapt figure received by ...]

While ST and context-based pedagogies share characteristics, there are important differences (Figure 5). ${ }^{28}$ While context-based learning has been shown to have benefits to chemistry education, ${ }^{39-43}$ encompassing more aspects of ST in the field of chemistry education may offer other distinct benefits for student learning as they are able to engage in more higherorder thinking skills, previously shown in other disciplines.

Our findings show that some educators primarily implement one aspect of STICE (i.e., teaching chemistry in context) which is considered the main similarity between STICE and context-based approaches (Figure 5). Therefore, efforts are needed to bridge knowledge of STICE and implementing STICE in their teaching. This knowledge gap may be addressed by clearly defining the criteria required to integrate STICE (e.g., what is required of the learner and instructor) as currently it remains unclear in the literature due to the vast range of definitions of STICE. ${ }^{35}$ Results of these efforts will help educators distinguish similar teaching approaches 
(e.g., context-based, problem-based, and inquiry-based learning, life cycle analysis, and multivariate reasoning) from STICE approaches, which will allow educators to identify the specific aspect of their current teaching that would need to be adapted to adopt a ST approach. Educators will then be able to better assess their feasibility and flexibility of integrating this approach to their course(s).

\section{Theme 4: Rating of barriers can guide priorities for educator/educational barriers}

Educators reported the extent of educator and educational barriers for implementing STICE into their course(s) (Figure 6). The weighted averages of each barrier show that educators' expertise on ST and barriers related to limited time and resources (i.e., limited time to learn and teach STICE, limited teaching resources and assessments) are perceived to have the greatest extent as barriers to STICE. Educators' expertise reported as a larger barrier indicates participants knowledge and/or experience may be a predictor for chemistry educators' willingness to implement STICE. Due to our limited sample size, we were unable to proceed with conducting a regression to test this hypothesis.

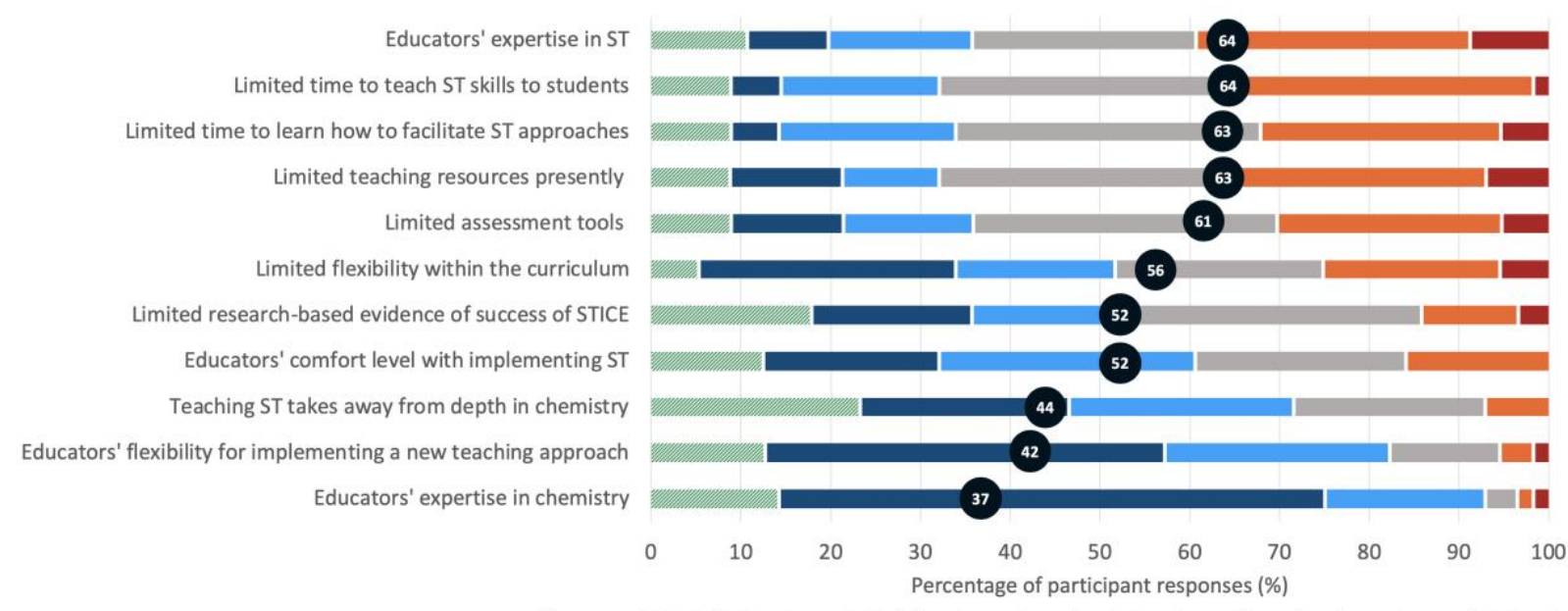

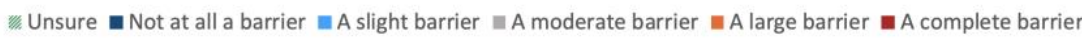

\# = Approximate weighted averages

Figure 6. The extent of educator and educational barriers for STICE self-reported by chemistry educators. Circled values represent weighted averages of the extent of each barrier; these are approximations as the data are categorical. ${ }^{44}$

Additionally, there is a need for these types of resources to be developed for a range of chemistry courses. One participant who creates their own learning activities stated they "spend a lot of time (and some internal funding) to develop new learning resources and learning activities." Minimizing barriers related to time to teach and learn STICE can be achieved with two key actions: (1) more openly available resources and (2) more effort to support the transition for educators to adopt this approach (e.g., professional development workshop and activities, textbooks, embedding STICE within curricula or guides to implementation). These actions will allow chemistry educators to spend less time trying to learn and develop their own resources, decreasing the extent of barrier towards implementing STICE. To date, there have been several webinars on STICE; ${ }^{45}$ however, educators report they want hands-on STICE experiences (SI, Table 6). 
Limited evidence-based research is considered a moderate barrier, understandable since little evidence is currently available about the effectiveness of the STICE approach. ${ }^{3}$ At the same time, chemistry educators may not know the extent of STICE literature available.

On the other hand, educators' flexibility for implementing STICE and educators' expertise in chemistry were mostly perceived as not being a barrier or slightly a barrier for enacting a STICE approach to their course(s). The reason why flexibility for implementing STICE may be perceived as less of a barrier is because generally, participants have a high level of flexibility for implementing new teaching approaches (SI, Figure 1).

Lastly, participants were most unsure about the lack of depth in chemistry as a barrier to STICE. This barrier relates to the perception that implementing STICE would come at the expense of depth of coverage of chemistry content. These educators might not have a clear understanding of how STICE might impact the depth of coverage of chemistry content due to the lack of educators' knowledge on STICE.

We hypothesize educators' level of experience with implementing STICE may influence the extent of each barrier. Two of the eleven educator/educational barriers showed a significant relationship with educators' experience with STICE. These educator barriers were: limited time to teach ST skills to students $\left(H(2)=7.504, p=0.023, E_{R}{ }^{2}=0.1501\right.$, medium effect size $)$ and educators' comfort level with implementing STICE $\left(H(2)=6.511, p=0.039, E_{R}^{2}=0.1356\right.$ (small to medium effect size)) (SI, Figure 10). In other words, once participants have experience with STICE, they may perceive barriers to have less of an impact than participants who have no experience with STICE.

Educational change models will likely help overcome these barriers to implementing STICE. A focus on educating educators on STICE may help educators understand how ST might impact the depth of content coverage in chemistry and might influence willingness to implement a STICE approach. Addressing contextual factors will allow educators to have teaching resources and assessment tools to be able to implement STICE and may increase their willingness to implement if STICE is integrated within curricula and evidence-based research shows it to be effective in specific learning contexts. Overall, efforts made towards educators' knowledge and contextual factors may result in more concerted efforts by chemistry educators to implement ST into their teaching practice.

\section{Conclusions}

To overcome educational challenges related to implementing a STICE approach, educators' perspectives and contexts need to be thoroughly understood in order to effectively address their needs. Educational change models can be adopted to help understand the interconnectedness of educator barriers and factors involved and guide future actions to address educator systemic change. We investigated chemistry educators' knowledge of and perspectives about STICE, in order to inform departmental, institutional, and administrative initiatives to implement STICE.

We found that educators' knowledge (i.e., low level of knowledge on STICE), contextual factors (i.e., willingness depends on availability of teaching resources, curriculum coverage, 
research-based evidence, and time constraints to learn and implement STICE) and personal factors (i.e., STICE approach needs to align with their teaching values or current teaching approach) all negatively influenced educators' willingness to implement a STICE approach. Therefore, efforts of change need to be made towards these three areas of the educator change model, to increase educators' willingness to adopt STICE approaches.

Educators reported experiences with STICE without knowing or specifically calling it ST; however, generally educators only consider that they implement STICE by teaching chemistry concepts and topics in context. Therefore, efforts need to be made to clearly distinguish the requirements of a STICE approach for the instructor and learner and how educators current teaching approaches (e.g., context-based learning) can be adapted to encompass the requirements of STICE.

Lastly, the rating of educator/educational barriers by participants indicates that some barriers related to educators' knowledge and contextual factors require more attention for change than other barriers.

Our results support the value of the TCSR model as a framework to develop instructional change for STICE and it may empower educational administrators and policy makers to consider the multifaceted nature of change when working with chemistry educators.

\section{Acknowledgements}

ARS thanks Canada's Social Science and Humanities Research Council (SSHRC) for funding through a Canada Graduate Scholarship-Doctoral scholarship.

\section{Author Contributions}

ARS and ABF conceived the project and designed the methods, in collaboration with PGM. ARS collected the data. ARS analysed the data with contributions from KB (qualitative data analysis) and $\mathrm{HL}$ (quantitative data analysis). All authors contributed to discussing results. ARS wrote the first draft of the manuscript. ABF and PGM edited the manuscript.

\section{Conflicts of interest}

The authors declare there were no conflicts of interest.

\section{References}

1. United Nations Sustainable Development Goals. (2015). Available at: http://sustainabledevelopment.un.org/. (Accessed: 11th November 2019)

2. Pazicni, S. \& Flynn, A. B. Systems Thinking in Chemistry Education: Theoretical Challenges and Opportunities. J. Chem. Educ. 96, 2752-2763 (2019).

3. Flynn, A. B. et al. Future Directions for Systems Thinking in Chemistry Education: Putting the Pieces Together. J. Chem. Educ. 96, 3000-3005 (2019).

4. Jerrentrup, R. The Effects of the Financial Crisis on the Future of the Chemical Industry. J. 
Bus. Chem. 6, 3-6 (2009).

5. Jun, Y. G. Friend or Enemy--Chemistry in Crisis (Chinese Edition). (Tianjin People's Publishing House, 2011).

6. Matlin, S. A., Mehta, G., Hopf, H. \& Krief, A. One-world chemistry and systems thinking. Nature Chemistry 8, 393-398 (2016).

7. Mahaffy, P. G., Krief, A., Hopf, H., Mehta, G. \& Matlin, S. A. Reorienting chemistry education through systems thinking. Nat. Rev. Chem. 2, 126 (2018).

8. Orgill, M., York, S. \& MacKellar, J. Introduction to Systems Thinking for the Chemistry Education Community. J. Chem. Educ. 96, 2720-2729 (2019).

9. Alien, R. T. Reductionism in Education. Philos. Inq. Educ. 5, 20-35 (1991).

10. Holme, T. A. \& Hutchison, J. E. A Central Learning Outcome for the Central Science. J. Chem. Educ. 95, 499-501 (2018).

11. Verhoeff, R. P., Knippels, M.-C. P. J., Gilissen, M. G. R. \& Boersma, K. T. The Theoretical Nature of Systems Thinking. Perspectives on Systems Thinking in Biology Education. Front. Educ. 3, 40 (2018).

12. Assaraf, O. B.-Z. \& Orion, N. Development of system thinking skills in the context of earth system education. J. Res. Sci. Teach. 42, 518-560 (2005).

13. Frank, M. Engineering systems thinking and systems thinking. Syst. Eng. 3, 163-168 (2000).

14. Lavi, R. \& Dori, Y. J. Systems thinking of pre- and in-service science and engineering teachers. Int. J. Sci. Educ. 41, 248-279 (2019).

15. Fanta, D., Braeutigam, J. \& Riess, W. Fostering systems thinking in student teachers of biology and geography - an intervention study. J. Biol. Educ. 54, 226-244 (2020).

16. Evagorou, M., Korfiatis, K., Nicolaou, C. \& Constantinou, C. An Investigation of the Potential of Interactive Simulations for Developing System Thinking Skills in Elementary School: A case study with fifth-graders and sixth-graders. Int. J. Sci. Educ. 31, 655-674 (2009).

17. Sabelli, N. H. Complexity, Technology, Science, and Education. J. Learn. Sci. 15, 5-9 (2006).

18. Aubrecht, K. B. et al. Graphical Tools for Conceptualizing Systems Thinking in Chemistry Education. J. Chem. Educ. 96, 2888-2900 (2019).

19. Chiu, M.-H., Mamlok-Naman, R. \& Apotheker, J. Identifying Systems Thinking Components in the School Science Curricular Standards of Four Countries. J. Chem. Educ. 96, 2814-2824 (2019).

20. Elmgren, M., Ho, F., Åkesson, E., Schmid, S. \& Towns, M. Comparison and Evaluation of Learning Outcomes from an International Perspective: Development of a Best-Practice Process. J. Chem. Educ. 92, 427-432 (2015). 
21. Lund, T. J. \& Stains, M. The importance of context: an exploration of factors influencing the adoption of student-centered teaching among chemistry, biology, and physics faculty. Int. J. STEM Educ. 2, 13 (2015).

22. Gess-Newsome, J., Southerland, S. A., Johnston, A. \& Woodbury, S. Educational Reform, Personal Practical Theories, and Dissatisfaction: The Anatomy of Change in College Science Teaching. Am. Educ. Res. J. 40, 731-767 (2003).

23. Stains, M. et al. Anatomy of STEM teaching in North American universities. Science 359, 1468-1470 (2018).

24. Woodbury, S. \& Gess-Newsome, J. Overcoming the paradox of change without difference: A model of change in the arena of fundamental school reform. Educ. Policy 16, 763-782 (2002).

25. Gibbons, R. E., Villafañe, S. M., Stains, M., Murphy, K. L. \& Raker, J. R. Beliefs about learning and enacted instructional practices: An investigation in postsecondary chemistry education. J. Res. Sci. Teach. 55, 1111-1133 (2018).

26. Popova, M., Shi, L., Harshman, J., Kraft, A. \& Stains, M. Untangling a complex relationship: Teaching beliefs and instructional practices of assistant chemistry faculty at research-intensive institutions. Chem. Educ. Res. Pract. 21, 513-527 (2020).

27. Mahaffy, P. G., Matlin, S. A., Holme, T. A. \& MacKellar, J. Systems thinking for education about the molecular basis of sustainability. Nat. Sustain. 2, 362-370 (2019).

28. York, S. \& Orgill, M. ChEMIST Table: A Tool for Designing or Modifying Instruction for a Systems Thinking Approach in Chemistry Education. J. Chem. Educ. 97, 2114-2129 (2020).

29. Lincoln, Y. S., Guba, E. G. \& Pilotta, J. J. Naturalistic inquiry. (1985).

30. Creswell, J.W., \& Miller, D. L. Determining Validity in Qualitative Inquiry. Theory Pract. 39, 124-130 (2000).

31. Field, A. Discovering Statistics Using IBM SPSS Statistics. (SAGE Publications, 2013).

32. Jackson, A. \& Hurst, G. A. Faculty perspectives regarding the integration of systems thinking into chemistry education. Chem. Educ. Res. Pract. 22, 855-865 (2021).

33. Schultz, M., Lai, J., Ferguson, J. P. \& Delaney, S. Topics Amenable to a Systems Thinking Approach: Secondary and Tertiary Perspectives. J. Chem. Educ. 98, 3100-3109 (2021).

34. Fairweather, J. S. Beyond the Rhetoric: Trends in the Relative Value of Teaching and Research in Faculty Salaries. J. Higher Educ. 76, 401-422 (2005).

35. Delaney, S., Ferguson, J. P. \& Schultz, M. Exploring opportunities to incorporate systems thinking into secondary and tertiary chemistry education through practitioner perspectives. Int. J. Sci. Educ. 1-22 (2021). doi:10.1080/09500693.2021.1980631

36. Weaver, G. C., Burgess, W. D., Childress, A. L. \& Slakey, L. Transforming institutions: Undergraduate STEM education for the 21st century. Purdue University Press (2015). 
37. Kezar, A. Higher Education Change and Social Networks: A Review of Research. J. Higher Educ. 85, 91-125 (2014).

38. Cohen, J. A Power Primer. Quant. Methods Psychol. 112, 155-159 (1992).

39. Broman, K. \& Parchmann, I. Students' application of chemical concepts when solving chemistry problems in different contexts. Chem. Educ. Res. Pr. 15, 516-529 (2014).

40. Parchmann, I. et al. "Chemie im Kontext": A symbiotic implementation of a contextbased teaching and learning approach. Int. J. Sci. Educ. 28, 1041-1062 (2006).

41. Overton, T. Context and problem-based learning. New Dir. Teach. Phys. Sci. 7-12 (2016). doi:10.29311/ndtps.v0i3.409

42. Overton, T. L. and Byers, B. and Seery, M. K. Context- and Problem-based Learning in Higher Level Chemistry Education. in Innovative Methods of Teaching and Learning Chemistry in Higher Education 45-61 (2009).

43. Gilbert, J. K. On the Nature of "Context" in Chemical Education. Int. J. Sci. Educ. 28, 957976 (2006).

44. Brown, J. D. Likert items and scales of measurement? SHIKEN JALT Test. Eval. SIG NewsI. 15, 10-14 (2011).

45. ACS Webinars. Available at: https://www.acs.org/content/acs/en/acs-webinars.html. (Accessed: 1st November 2021) 